\title{
Characterization of volatile compounds in fermented milk using solid-phase microextraction methods coupled with gas chromatography-mass spectrometry
}

\author{
T. Dan, ${ }^{1}$ D. Wang, ${ }^{1}$ R. L. Jin, H. P. Zhang, T. T. Zhou, and T. S. Sun ${ }^{2}$ \\ Key Laboratory of Dairy Biotechnology and Engineering, Ministry of Education, Inner Mongolia Agricultural University, Hohhot, 010018, \\ P. R. China
}

\begin{abstract}
Lactic acid bacteria (LAB) are industrially important bacteria that are widely used in the fermented food industry, especially in the manufacture of yogurt. Characteristic flavors are produced by LAB during fermentation and storage that affect the quality and acceptability of fermented milk products. In this study, the volatile compounds in milk fermented by Streptococcus thermophilus IMAU80842 alone, Lactobacillus delbrueckii ssp. bulgaricus IMAU20401 alone, or both species together were identified using solid-phase microextraction methods coupled with gas chromatographymass spectrometry. A total of 53,43 , and 32 volatile compounds were identified in milk fermented by $S$. thermophilus alone, L. delbrueckii ssp. bulgaricus alone, or both species together, respectively. The presence of some important flavor compounds was confirmed: acetic acid, acetaldehyde, acetoin, 2,3-butanedione, ethanol, and 1-heptanol. Our results demonstrate that the composition of the volatile compounds in fermented milk depends on the species of LAB used and whether they are used alone or in combination. This is important for the selection of appropriate starter cultures for the production of different types of fermented milk product with particular flavors.
\end{abstract}

Key words: fermented milk, volatile compounds, solidphase microextraction methods, gas chromatography/ mass spectrometry

\section{INTRODUCTION}

Lactic acid bacteria (LAB) are industrially important bacteria that are widely used in the fermented food industry, especially in the manufacture of yogurt.

Received May 30, 2016.

Accepted December 12, 2016.

${ }^{1}$ T. Dan and D. Wang contributed equally to this study.

${ }^{2}$ Corresponding author: sts9940@sina.com
Yogurt is the most popular fermented milk, and usually produced using mixtures of homofermentative LAB such as Streptococcus thermophilus and Lactobacillus delbrueckii ssp. bulgaricus as the starter culture (HerveJimenez et al., 2009; Muramalla and Aryana, 2011; Kaneko et al., 2014).

During fermentation, S. thermophilus and L. delbrueckii ssp. bulgaricus generate lactic acid and a variety of volatile organic aroma compounds. These compounds impart particular flavors to fermented milk (Beshkova et al., 1998). The overall flavor of any fermented milk product is formed by a large number of these volatile compounds. Among these compounds, carbonyl compounds and organic acids such as acetaldehyde, diacetyl, and acetic acid play a crucial role and can be used to evaluate the flavor quality of dairy products (Zha et al., 2015). Ott et al. (1997) found that acetaldehyde provided the typical flavor of dairy products such as yogurt and buttermilk. Erkus et al. (2013) also reported that diacetyl contributed the characteristic sour buttery flavor to some fermented dairy products.

Solid-phase microextraction methods (SPME) were developed in the 1990s by Arthur and Pawliszyn (1990) as a fast and useful technique for the analysis of volatile compounds. The SPME methods coupled with GC-MS can provide high sensitivity with a small sample volume; therefore, it can be used to analyze the flavor profile of a wide variety of substances. Most recently, this technique has been used to study the volatile profiles of fermented camel milk (Ning et al., 2011), grapes and wine (Panighel and Flamini, 2014), and dry fermented sausage (Corral et al., 2016).

In this study, SPME pretreatment coupled with GC-MS was used to study the volatile compounds in fermented milk compounds during fermentation and storage. The aim of this study was to investigate the composition of the volatile compounds in milk that had been fermented by either $S$. thermophilus IMAU 80842 alone, L. delbrueckii ssp. bulgaricus IMAU20401 alone, or both species together. 


\section{MATERIALS AND METHODS}

\section{Sample Preparation}

Sterile milk was prepared by reconstituting $10 \%$ (wt/ vol) skimmed milk powder in distilled water and autoclaving at $95^{\circ} \mathrm{C}$ for $5 \mathrm{~min}$. It was stored at $4^{\circ} \mathrm{C}$ before use.

The S. thermophilus IMAU80842 and L. delbrueckii ssp. bulgaricus IMAU20401 isolates from the Lactic Acid Bacteria Collection Center of Inner Mongolia Agricultural University were used throughout this study. These isolates were from traditionally produced yogurt and kurut from Mongolia and from the Gansu province of China. Frozen cells of these isolates were activated by 3 subcultures using de Man, Rogosa, Sharpe broth (Becton, Dickinson and Co., Sparks, MD) and then inoculated into $100 \mathrm{~mL}$ of reconstituted $10 \%$ (wt/vol) milk medium and incubated for $24 \mathrm{~h}$ at $42^{\circ} \mathrm{C}$. Inoculations were made to achieve a final concentration of approximately $5 \times 10^{6} \mathrm{cfu} / \mathrm{mL}$. Three inoculation treatments were made: pure $S$. thermophilus, pure L. delbrueckii ssp. bulgaricus, and a 1:1 mixture of $S$. thermophilus and L. delbrueckii ssp. bulgaricus. After inoculation, the milk was fermented at $42^{\circ} \mathrm{C}$ until the $\mathrm{pH}$ value fell to 4.5 , and was then stored at $4^{\circ} \mathrm{C}$. Samples were taken from each culture after $0,1,3,7$, and $14 \mathrm{~d}$ during the storage period. The samples collected were stored at $-20^{\circ} \mathrm{C}$ until the volatile compounds were analyzed.

\section{Isolation of Volatile Compounds}

Volatile compounds from the fermented milk were isolated using the headspace SPME technique (Ning et al., 2011). All extractions were performed using 50/30 $\mu \mathrm{m}$ divinylbenzene/carboxen/polydimethylsiloxane fibers. The SPME fiber was purchased from Supelco Inc. (Bellefonte, PA). Five-milliliter samples of each of the fermented milk products were placed into $15-\mathrm{mL}$ glass vials (Supelco Inc.) with micro-stirring bars and stirred for $60 \mathrm{~min}$ at $55^{\circ} \mathrm{C}$ to allow the samples to reach equilibrium. The fiber was inserted into the injection port of the Agilent 7890B gas chromatograph (Agilent Technologies Inc., Palo Alto, CA), held for 5 min for preconditioning, and then inserted in to the vial and exposed in the headspace for $60 \mathrm{~min}$ under the above conditions. After absorbing the volatile compounds, the fibers were inserted into the GC-MS injector port for desorption $(3 \mathrm{~min})$ at $270^{\circ} \mathrm{C}$ to desorb volatile compounds into the gas chromatograph.

Volatile compounds from the fermented milk were identified using an 7890B gas chromatograph equipped with an 5977A mass selective detector (both from Agilent Technologies Inc.). Volatile compounds absorbed onto the SPME fiber were passed through an HP-5MS column (30 m length, $0.25 \mathrm{~mm}$ inside diameter, $0.25 \mu \mathrm{m}$ film thickness; Agilent Technologies Inc.) with helium as the carrier gas at $1 \mathrm{~mL} / \mathrm{min}$. The gas chromatograph temperature was maintained at $35^{\circ} \mathrm{C}$ for $5 \mathrm{~min}$, then increased to $140^{\circ} \mathrm{C}$ at a rate of $4^{\circ} \mathrm{C} / \mathrm{min}$ for $5 \mathrm{~min}$, and finally gradually increased to $250^{\circ} \mathrm{C}$ at a rate of $10^{\circ} \mathrm{C} /$ min for $5 \mathrm{~min}$. The transfer line temperature was set to $250^{\circ} \mathrm{C}$. The mass detector was operated at $150^{\circ} \mathrm{C}$ in electron impact mode at a voltage of $70 \mathrm{eV}$ and an ion source temperature of $230^{\circ} \mathrm{C}$. Mass spectra of different treated samples were recorded with a mass range of 40 to $400 \mathrm{~m} / \mathrm{z}$, with 5 scans and no solvent delay.

\section{Identification of Volatile Compounds}

Volatile compounds were identified by comparing their mass spectra with those from a published database (NIST version 11 mass spectral database; Agilent Technologies Inc.). To calculate the retention indices (RI) of detected compounds by the NIST 11 database in the same capillary column, a series of $n$-alkanes C3-C25 (AccuStandard Inc., New Haven, CT) were run under the same chromatographic conditions. Furthermore, acetaldehyde, hydroxyacetic acid, acetic acid, 2,3-butanedione, ethanol, propylene glycol, formic acid ethenyl ester, and acetic acid ethenyl ester were used as standards to confirm the identifications. Acetaldehyde, hydroxyacetic acid (99.5\%), acetic acid (99.9\%), and ethanol (99.9\%) were obtained from Dr. Ehrenstorfer GmbH (Augsburg, Germany), and the other compounds were obtained from Sigma-Aldrich (Steinheim, Germany).

\section{RESULTS AND DISCUSSION}

Volatile compounds from fermented milk are very diverse and have an effect on flavor. We used SPME GC-MS techniques to compare the volatile flavor compounds of fermented milk from pure cultures and mixed cultures. Results of volatile compounds profile and their relative contents are summarized in Tables 1 to 6 .

\section{Separation of Acids}

Volatile acid compounds extracted with SPME and analyzed by GC-MS were from different fermented milks produced by $S$. thermophilus and L. delbrueckii ssp. bulgaricus that were cultured alone or as a mixture. Seven acid compounds were identified in the volatile fraction of $S$. thermophilus-fermented milk (Table 1). The quantity of each acid compound increased after $1 \mathrm{~d}$ of storage. This increase was particularly evident for acetic acid, 4-chlorobutanoic acid, 3-methylbutanoic 
acid, hexanoic acid, octanoic acid, and $n$-decanoic acid; these acid compounds all contribute good flavor to fermented milk.

Nine volatile acid compounds were detected in the volatile fraction from L. delbrueckii ssp. bulgaricusfermented milk (Table 1), but only 5 were the same as those produced by $S$. thermophilus (i.e., acetic acid, 3-methylbutanoic acid, hexanoic acid, octanoic acid, and $n$-decanoic acid). Generally, the quantity of each acid compound increased after $3 \mathrm{~d}$ of storage. This increase was particularly obvious for acetic acid, butanoic acid, and heptanoic acid.

Nine volatile acid compounds were detected in the volatile fraction of fermented milk produced by a mixture of S. thermophilus and L. delbrueckii ssp. bulgaricus (Table 1). Among these acid compounds, 5 were also detected in fermented milk produced by one or other of these LAB in pure culture: acetic acid, butanoic acid, 3-methylbutanoic acid, hexanoic acid, and octanoic acid. The quantity of each acid compound increased after either 3 or $7 \mathrm{~d}$ of storage, depending on the compound. This increase was particularly obvious for acetic acid and heptanoic acid.

Acetic acid has been found previously as a volatile in yogurt by Panagiotidis et al. (2001). High levels of acetic acid contributes most to the tart flavor of yogurt and is particularly associated with a "vinegary, pungent, acidic" taste (Panagiotidis et al., 2001). In this study, high levels of acetic acid were detected in fermented milk produced by both pure and mixed cultures. The quantity of acetic acid in L. delbrueckii ssp. bulgaricus-fermented milk and mixed cultures was significantly higher than from milk fermented by $S$. thermophilus alone, and ranged from 3.48 to $16.82 \%$ and 8.47 to $25.92 \%$ of all volatile compounds after 14 d storage at $4^{\circ} \mathrm{C}$, respectively. These results are in accordance with Corcoran et al. (2005) and Ongol et al. (2007), who showed that L. delbrueckii ssp. bulgaricus itself is highly resistant to lactic acid and continues to ferment lactose to lactic acid in a low-pH environment, even after fermentation.

Besides acetic acid, hexanoic acid is a prime source of flavor and functionality in fermented milk. Hexanoic acid increases the odor of yogurt and contributes to the "pungent, rancid, flowery" flavor as found previously by Pereda et al. (2008), who detected hexanoic acid using divinylbenzene/carboxen/polydimethylsiloxane fibers in ultra-high-pressure homogenized milk. During storage, we detected hexanoic acid in fermented milk produced by both the pure and mixed cultures. As with acetic acid, the quantity of hexanoic acid in milk fermented by $S$. thermophilus alone was significantly lower than in milk fermented by $L$. delbrueckii ssp. bulgaricus alone or the mixed culture. This indicates that L. delbrueckii ssp. bulgaricus IMAU20401 may be an acid-tolerant bacterium that continues to produce hexanoic acid in a low-pH environment during storage.

In contrast to hexanoic acid and acetic acid, lower levels of 3-methylbutanoic acid, cyclohexanecarboxylic acid, and $n$-decanoic acid were detected in both pure and mixed-culture fermented milk. Although these were in lower quantities than acetic acid and hexanoic acid, they still play specific roles in the aroma and flavor properties of fermented milk (Pogačić et al., 2016).

Lactobacillus delbrueckii ssp. bulgaricus and S. thermophilus can generate many metabolic products even after fermentation has finished [e.g., the lactose present in the milk is converted to acid compounds during storage (Rascón-Díaz et al., 2010)]. This may be due to the occurrence of postacidification, which results in acid compounds increasing during storage and after fermentation.

\section{Separation of Aldehydes}

Six aldehyde compounds were detected in the volatile fraction of $S$. thermophilus-fermented milk (Table 2). Acetaldehyde is an indispensable carbonyl component of fermented milk and sufficient levels are required to impart an "ethereal, fresh, green, pungent" flavor to yogurt (Georgala et al., 1995; Gaafar, 2007). Acetaldehyde is produced by various LAB including $S$. thermophilus and L. delbrueckii ssp. bulgaricus (Bodyfelt and Potter, 2008; Akyol et al., 2015; Gezginc et al., 2015). In our study the quantity of acetaldehyde in milk fermented by $S$. thermophilus increased significantly after 0 or $1 \mathrm{~d}$ of storage, peaking at $6.61 \%$ of all volatile compounds. Besides acetaldehyde, the quantity of benzaldehyde in milk fermented by $S$. thermophilus also increased after $1 \mathrm{~d}$ of storage at $4^{\circ} \mathrm{C}$, peaking at $1.12 \%$ of all volatile compounds. Benzaldehyde can impart a unique flavor to fermented milk (e.g., it gives an aromatic note of bitter almonds at low levels and maraschino cherries at higher levels; Buttery et al., 1988). Benzaldehyde is the predominant aldehyde detected in certain dairy products such as Camembert cheese (Dumont et al., 1974a,b). Heptanal is also an important aroma compound that gives the "green, sweet" flavor to yogurt (Cheng, 2010). Even at low levels, heptanal can increase the flavor quality of dairy products (Siek et al., 2006). A low level of heptanal was detected in S. thermophilusfermented milk, ranging from 0.21 to $0.6 \%$ of all volatile compounds after $14 \mathrm{~d}$ of storage. Similar results have been reported by Condurso et al. (2008), where a trace concentration of heptanal was detected in fresh goat cheese.

Ten aldehyde compounds were detected in the volatile fraction of milk fermented by L. delbrueckii ssp. bulgari- 


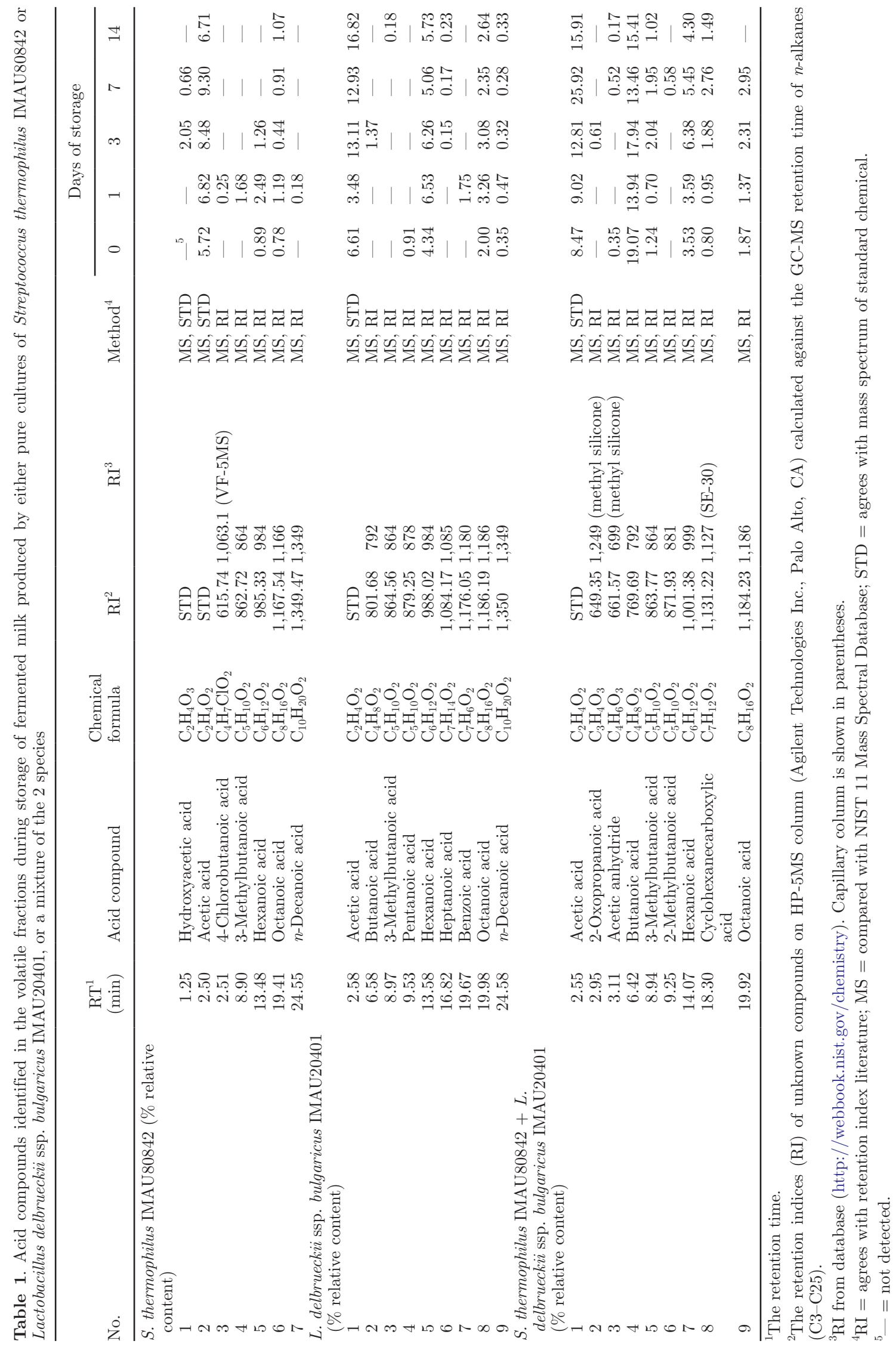


DAN ET AL.

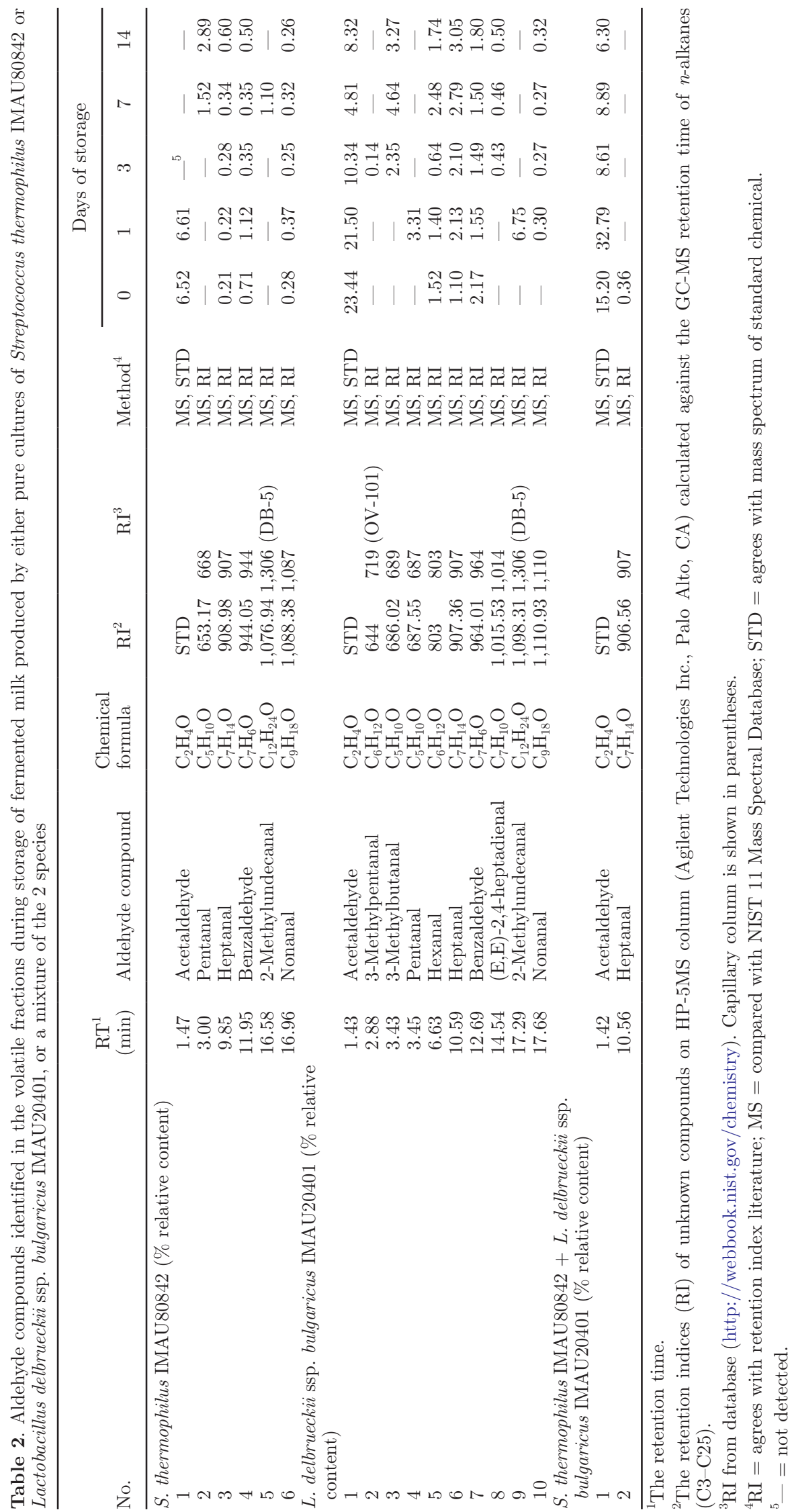


cus (Table 2). Among these aldehyde compounds, 6 were also detected in the volatile fraction of milk fermented by $S$. thermophilus: acetaldehyde, benzaldehyde, heptanal, nonanal, pentanal, and 2-methylundecanal. The remaining 4 aldehyde compounds were not detected in the volatile profile of $S$. thermophilus-fermented milk. The quantity of acetaldehyde, pentanal, and 2-methylundecanal increased significantly after 0 to $1 \mathrm{~d}$ of storage and was particularly obvious for acetaldehyde which reached $23.44 \%$ of all volatile compounds after $0 \mathrm{~d}$ of storage. Hexanal is also an important aldehyde that contributes to the "green, cut-grass" flavor of yogurt (Cheng, 2010). In our study, hexanal was detected in the L. delbrueckii ssp. bulgaricus-fermented milk during storage, and peaked at $2.48 \%$ of all volatile compounds after $7 \mathrm{~d}$ of storage, falling to $1.74 \%$ by $\mathrm{d}$ 14. Similar results were reported by Margalith (1981). Hexanal is the main aldehyde present in Camembert cheese (Dumont et al., 1974b).

Only 2 aldehyde components were detected in the volatile fraction of the fermented milk produced by a mixture of $S$. thermophilus and L. delbrueckii ssp. bulgaricus (Table 2). The quantity of acetaldehyde was greater than the other aldehyde components, and ranged from 6.3 to $32.79 \%$ of all volatile compounds after $14 \mathrm{~d}$ of storage at $4^{\circ} \mathrm{C}$, peaking at $32.79 \%$ within 1 $\mathrm{d}$ of storage and declining thereafter. Meanwhile, only low relative content (RC) values of heptanal were detected, peaking at just $0.36 \%$ of all volatile compounds after $1 \mathrm{~d}$ of storage.

\section{Separation of Ketones}

Ten ketone compounds were detected in the volatile fraction from S. thermophilus-fermented milk (Table 3). Among these ketones, the main ketone compounds were 2,3-butanedione, acetoin, 2-heptanone, and 2-nonanone. These compounds were present at high levels. For example, levels of 2,3-butanedione and acetoin ranged from 3.54 to $11.62 \%$ and 8.25 to $20.36 \%$, respectively, after $14 \mathrm{~d}$ of storage. Both of these compounds are obtained from pyruvate, which comes from lactose and citrate metabolism (Starrenburg and Hugenholtz, 1991; Pan et al., 2014) and imparts a "buttery, creamy, vanilla" flavor to fermented milk (Settachaimongkon et al., 2014). These 2 compounds have been reported by Ott et al. (1997), Beshkova et al. (2003), and Chaves et al. (2011) as important flavor compounds in fermented milk. Our observations are in accordance with theirs, though we detected a higher content of 2,3-butanedione. 2,3-Butanedione is a diketone and can readily be converted to acetoin by the enzyme diacetyl reductase (Collins, 1972; Carballo et al., 1991; Rattray et al., 2003). The flavor characteristics of 2,3-butanedione and acetoin are similar and in combination contribute to the "buttery" flavor of fermented milk. These 2 compounds are known to influence the aroma and flavor qualities of fermented milk (Nieto-Arribas et al., 2011).

A total of 8 ketone compounds were detected in the volatile fraction of $L$. delbrueckii ssp. bulgaricusfermented milk (Table 3). However, the important flavor compound, 2,3-butanedione, which was found from $S$. thermophilus-fermented milk, was not detected. Furthermore, only low RC values of acetoin were detected peaking at only $2.53 \%$ of all volatile compounds after $3 \mathrm{~d}$ of storage, and declining to $0.23 \%$ of all volatile compounds after $7 \mathrm{~d}$. In the volatile fraction of $L$. delbrueckii ssp. bulgaricus-fermented milk, 2-heptanone and 2-nonanone were the predominant ketone compounds. The quantity of 2-heptanone and 2-nonanone ranged from 9.92 to $14.47 \%$ and 4.24 to $7.29 \%$ of all volatile compounds after $14 \mathrm{~d}$ of storage, respectively; the highest levels were found at $0 \mathrm{~d}$, so levels declined with storage. 2-Heptanone and 2-nonanone are reported as key aroma compounds that contribute to the "creamy, fresh" flavor of fermented milk (Beshkova et al., 2003; Pionnier and Hugelshofer, 2006). These compounds have been reported previously from dairy products (Pionnier and Hugelshofer, 2006).

Six ketone compounds were detected in the volatile fraction of the fermented milk made by a mixture of $S$. thermophilus and L. delbrueckii ssp. bulgaricus. This was similar to the ketones found in milk fermented by L. delbrueckii ssp. bulgaricus alone, with only trace amounts of 2,3-butanedione. Higher quantities of 2-pentanone, 2-heptanone, and 2-nonanone were detected from the mixed culture than from milk fermented by L. delbrueckii ssp. bulgaricus alone. In particular, 2-heptanone ranged from 6.63 to $20.72 \%$ of all volatile compounds after $14 \mathrm{~d}$ of storage, though the highest levels were found at $0 \mathrm{~d}$ and so declined during storage.

\section{Separation of Alcohols}

A total of 9 alcohol compounds were identified in the volatile fraction from $S$. thermophilus-fermented milk (Table 4). Ethanol is a common fermentation product for many LAB and is produced by reduction of acetaldehyde (de Vos and Hugenholtz, 2004). High levels of ethanol were produced from milk fermented by $S$. thermophilus throughout storage; levels ranged between 8.13 and $10.99 \%$. As mentioned previously, the quantity of acetaldehyde was greatest at 0 and $1 \mathrm{~d}$ of storage and no acetaldehyde was detected after 3,7 , and 14 d. These results indicated that adh (coding for alcohol dehydrogenase) may be present in the $S$. thermophilus IMAU80842 genome. This supports the observation of high levels of ethanol, which would result from overex- 
DAN ET AL.

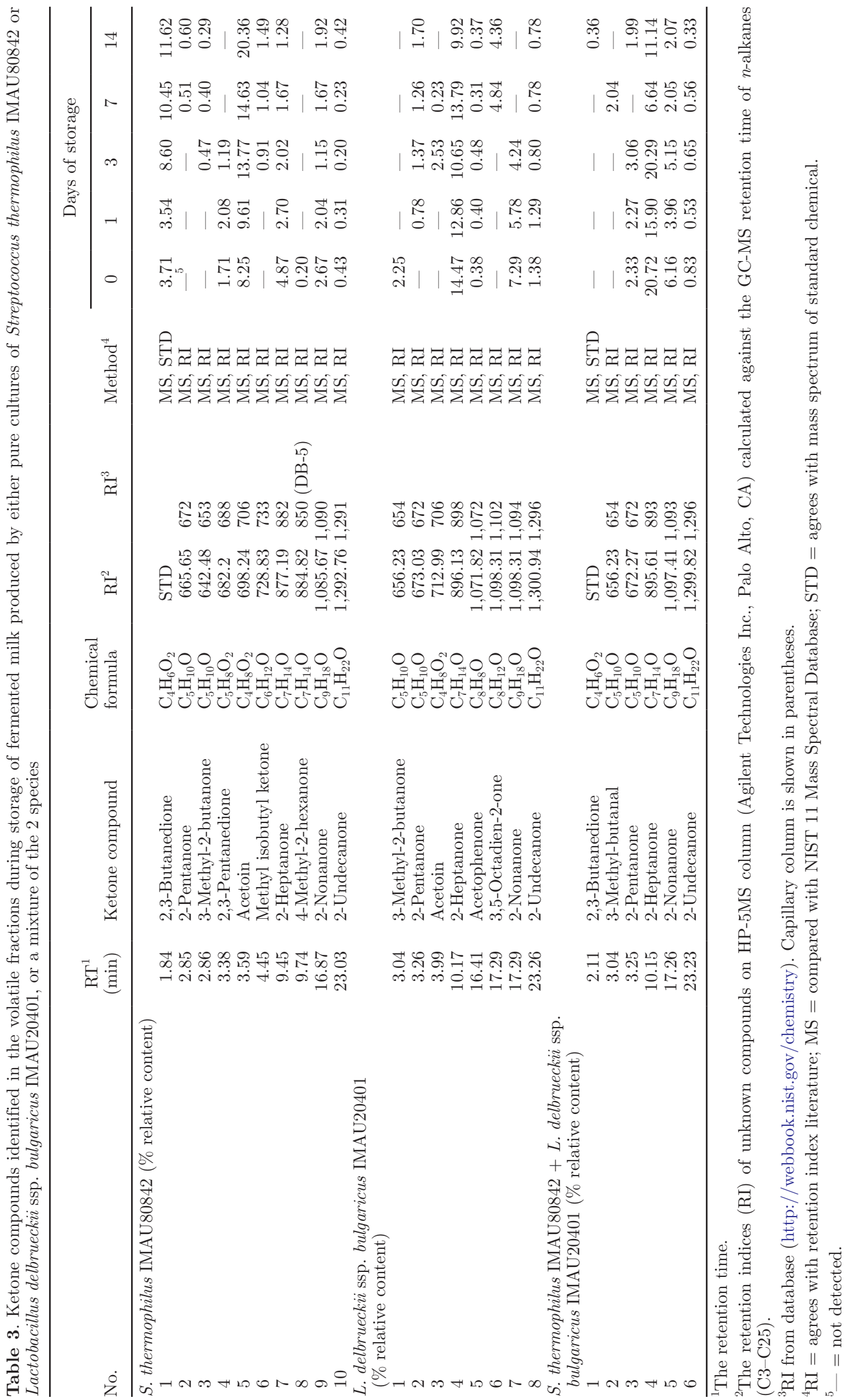


pression of the $a d h B$ gene (Akyol et al., 2015). Beside ethanol, the other main alcohols were 1-hexanol and 1-heptanol, both of which are also considered as important flavor compounds in dairy products (Dumont et al., 1974b).

A total of 8 alcohol compounds were detected in the volatile fraction from $L$. delbrueckii ssp. bulgaricusfermented milk (Table 4). Among these, 1-hexanol and 1-heptanol were present at the highest levels ranging from 3 to $3.21 \%$ and 4.44 to $5.91 \%$ of all volatile compounds, respectively. In contrast to the volatile fraction from $S$. thermophilus-fermented milk, no ethanol was detected in the volatile fraction from $L$. delbrueckii ssp. bulgaricus. As mentioned previously, acetaldehyde content increased during storage of milk fermented by $L$. delbrueckii ssp. bulgaricus, and this suggests that adh may not be present in the L. delbrueckii ssp. bulgaricus IMAU20401 genome.

A total of 8 alcohol compounds were detected in the volatile fraction of the fermented milk made by a mixture of $S$. thermophilus and L. delbrueckii ssp. bulgaricus (Table 4). Only low levels of alcohol compounds were detected in this sample. Among these the levels of 1-hexanol, 1-heptanol, and 2-nonanol did not differ during storage, ranging from 0.35 to $0.54 \%, 0.44$ to $0.73 \%$, and 0.29 to $0.73 \%$ of all volatile compounds, respectively.

\section{Separation of Esters}

Even at low concentrations ester compounds contribute to the "fruity and floral" aromas and flavors of dairy products such as yogurt (Guler and Stupp, 2007). A total of 6 ester compounds were detected in the volatile fraction from milk fermented by $S$. thermophilus alone (Table 5). Among these, high $\mathrm{RC}$ values were detected for butanoic acid ethyl ester, ranging from 3.2 to $4.12 \%$ of all volatile compounds after $14 \mathrm{~d}$ of storage. This is similar to the results reported by Pan et al. (2014) who detected butanoic acid methyl ester using SPME GC-MS. Besides butanoic acid ethyl ester, some other flavor compounds such as acetic acid butyl ester, decanoic acid ethyl ester, and $\delta$-nonalactone were also found in the sample. Low RC values were detected for decanoic acid ethyl ester after $1 \mathrm{~d}$ of storage, which has been reported previously by Ning et al. (2011).

Only 2 ester compounds were detected in the volatile fraction from $L$. delbrueckii ssp. bulgaricus-fermented milk. Among these, formic acid ethenyl ester achieved the highest levels, ranging from 2.56 to $10.45 \%$ of all volatile compounds after $14 \mathrm{~d}$ of storage; highest levels were achieved after $3 \mathrm{~d}$ of storage. Other flavor compounds, such as butanoic acid ethyl ester, were found in the sample but only at low levels. Even at low levels, these compounds are essential for the formation of flavor in fermented milk.

A total of 3 ester compounds were found in the volatile fraction of the fermented milk made by a mixture of S. thermophilus and L. delbrueckii ssp. bulgaricus (Table 5). Among these, formic acid ethenyl ester was present at higher levels in the volatile fraction from the mixed culture than from milk fermented by L. delbrueckii ssp. bulgaricus alone. In the mixed culture, this compound peaked at $11.81 \%$ of all volatile compounds after 14 d of storage, although it was not found after 3 or $7 \mathrm{~d}$ of storage. Acetic acid ethenyl ester was also found at higher levels in the volatile fraction from the mixed culture, ranging from 0.87 to $7.28 \%$, compared with the volatile fraction from milk fermented by $L$. delbrueckii ssp. bulgaricus alone. Similarly, for the volatile fraction from $S$. thermophilus-fermented milk, only low RC values were detected for $\delta$-nonalactone after 0,1 , and $3 \mathrm{~d}$ of storage.

\section{Separation of Hydrocarbons}

A total of 15 hydrocarbon compounds were found in the volatile fraction of $S$. thermophilus-fermented milk (Table 6). Among these compounds, high levels of 1,3,5-cycloheptatriene were present, ranging from 0.46 to $4.06 \%$ of all volatile compounds after $14 \mathrm{~d}$ of storage. The other main hydrocarbon compound present was $p$-xylene, which ranged from 0.83 to $1.82 \%$ of all volatile compounds produced during storage. Similar results have been reported from fresh goat cheese by Condurso et al. (2008), where the quantity of $p$-xylene was constant throughout the $21 \mathrm{~d}$ of storage.

A total of 6 hydrocarbon compounds were found in the volatile fraction from milk fermented by $L$. delbrueckii ssp. bulgaricus alone (Table 6). The quantity of these compounds was lower than from milk fermented by $S$. thermophilus alone. The quantity of 2-nonyne and 1-undecyne were the highest, reaching 2.58 and $2.04 \%$ of all volatile compounds after 0 and $14 \mathrm{~d}$ of storage, respectively. Levels of $p$-xylene were lower in the volatile fraction from $L$. delbruekii ssp. bulgaricus than in the fraction from $S$. thermophilus-fermented milk, and were similar throughout the storage period ranging from 0.17 to $0.37 \%$ of all volatile compounds.

The hydrocarbon compounds in the volatile fraction of the fermented milk made by a mixture of $S$. thermophilus and L. delbrueckii ssp. bulgaricus were different from the volatile profiles of both $S$. thermophilus and L. delbrueckii ssp. bulgaricus when they were cultured individually. Only 4 compounds were detected. Moreover, only low levels of toluene and $p$-xylene were detected from the mixed culture, and were lower than those of both the pure cultures. Toluene and $p$-xylene, 
DAN ET AL.

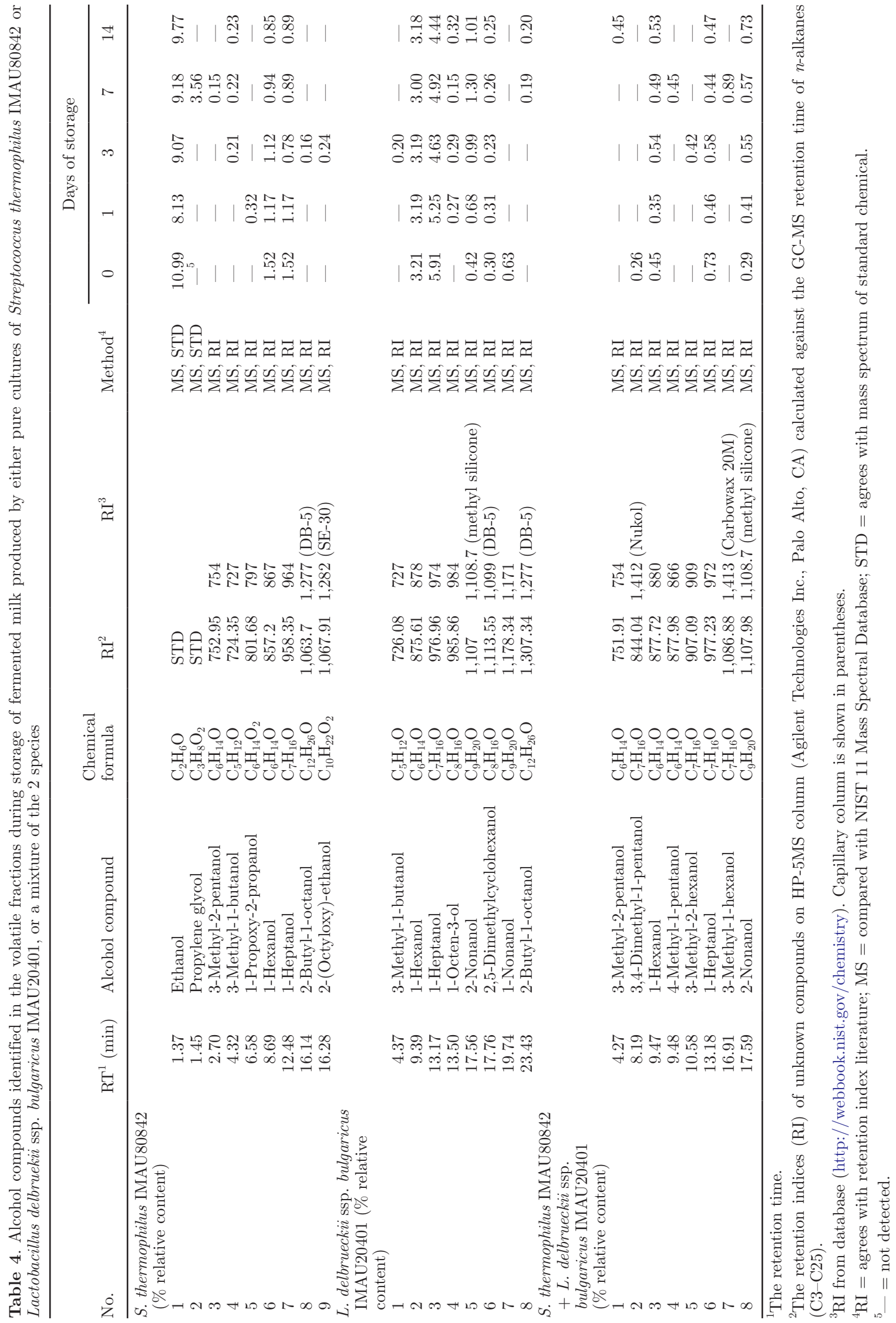


CHARACTERIZATION OF VOLATILE COMPOUNDS

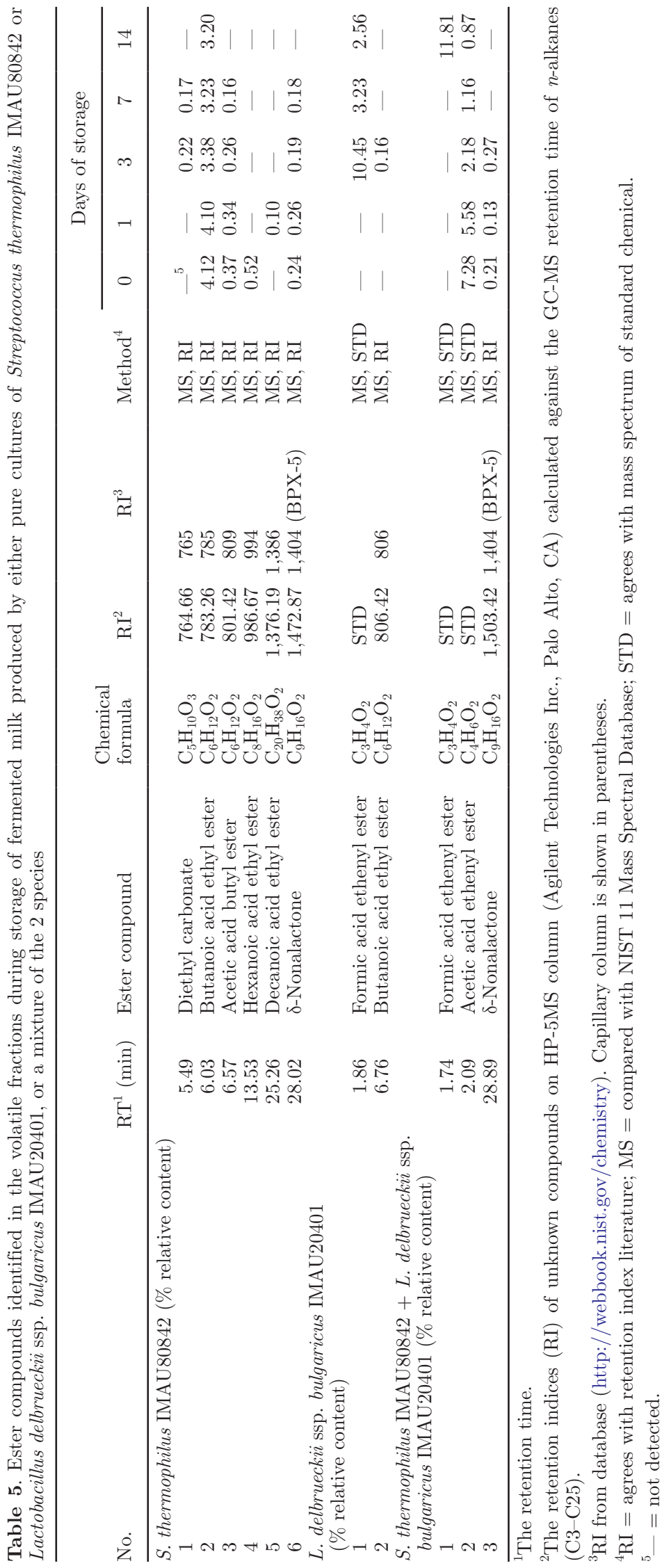


DAN ET AL.

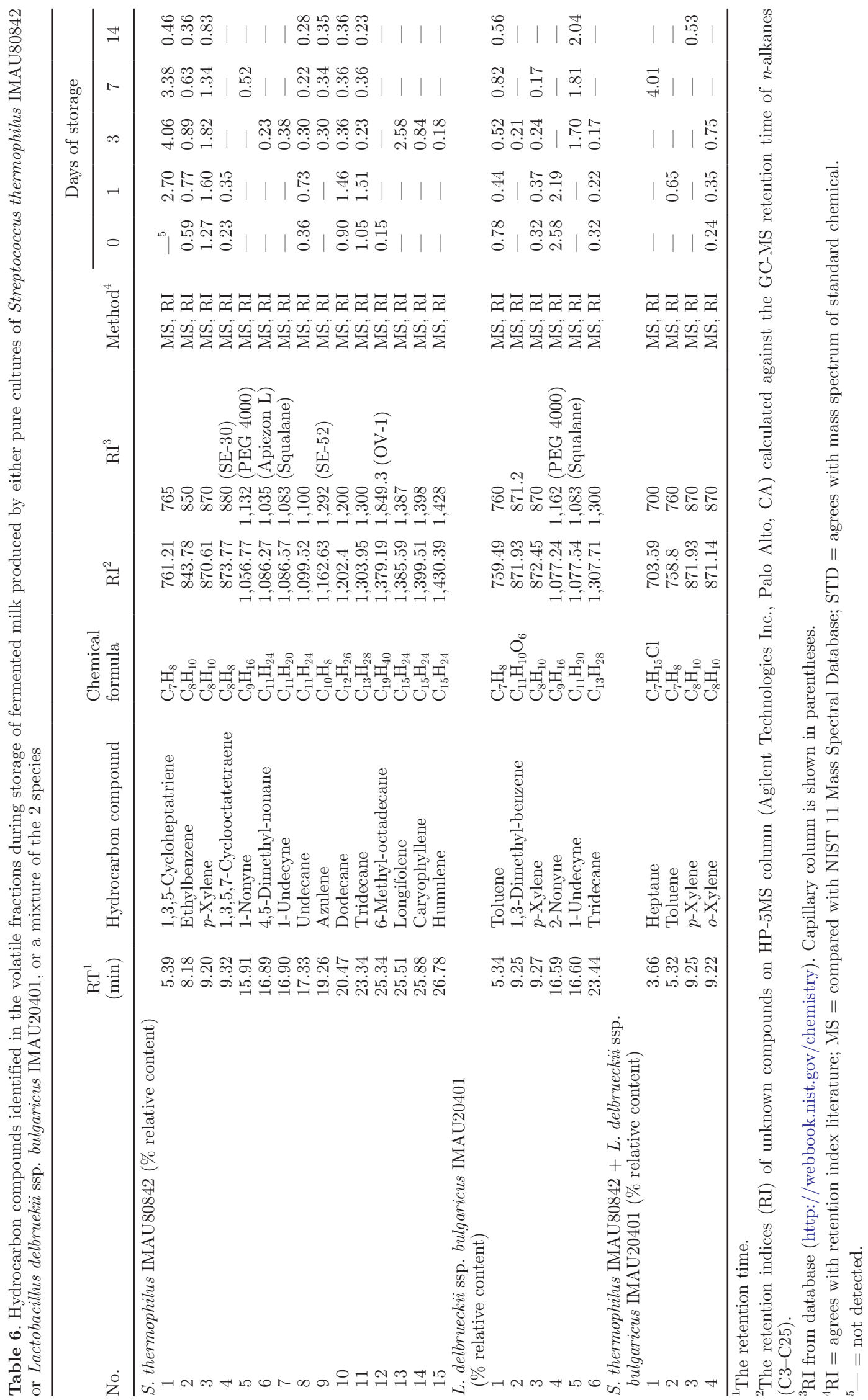


as volatile compounds, have been reported previously by Condurso et al. (2008).

\section{CONCLUSIONS}

In this study, the volatile compounds from fermented milk were isolated by SPME methods and were analyzed by GC-MS. Many compounds were identified in the different samples. A total of 53 volatile compounds were identified in the volatile fraction from $S$. thermophilusfermented milk, which included 7 acids, 6 aldehydes, 10 ketones, 9 alcohols, 6 esters, and 15 hydrocarbon compounds; among these, the major volatile compounds were acetic acid, acetaldehyde, 2,3-butanedione, acetoin, ethanol, and butanoic acid ethyl ester. A total of 43 volatile compounds were identified in the volatile fraction of L. delbrueckii ssp. bulgaricus-fermented milk, which included 9 acids, 10 aldehydes, 8 ketones, 8 alcohols, 2 esters, and 6 hydrocarbon compounds; among these, the major volatile compounds were acetic acid, hexanoic acid, acetaldehyde, 2-heptanone, 1-heptanol, and formic acid ethenyl ester. A total of only 32 volatile compounds were identified from the volatile fraction of the fermented milk by a mixture of $S$. thermophilus and L. delbrueckii ssp. bulgaricus, and included 9 acids, 2 aldehydes, 6 ketones, 8 alcohols, 3 esters, and 4 hydrocarbon compounds; the major volatile compounds were acetic acid, butanoic acid, acetaldehyde, 2-heptanone, 2-nonanone, and acetic acid ethenyl ester. In conclusion, the volatiles produced from pure cultures and mixtures were different, including variation in some important flavor compounds known to provide the characteristic flavor of different fermented milk products. These results will inform the selection of pure or mixed cultures for the production of fermented milk products with particular flavors. These results also demonstrate that the SPME technique was a useful tool in detecting the volatile compounds produced by fermented milk.

\section{ACKNOWLEDGMENTS}

This research was supported by National Natural Science Foundation of China (Beijing; No. 31460446, $31471711)$.

\section{REFERENCES}

Akyol, I., F. G. Ozcelik, A. Karakas-Sen, E. Ozkose, Y. Gezginc, and M. S. Ekinci. 2015. Cloning and overexpression of the als, pfla, and adhb genes in Streptococcus thermophilus and their effects on metabolite formation. Mol. Biotechnol. 57:923-930.

Arthur, C. L., and J. Pawliszyn. 1990. Solid phase microextraction with thermal desorption using fused silica optical fibers. Anal. Chem. 62:2145-2148.
Beshkova, D., E. Simova, G. Frengova, and Z. Simov. 1998. Production of flavor compounds by yogurt starter cultures. J. Ind. Microbiol. Biotechnol. 20:180-186.

Beshkova, D. M., E. D. Simova, G. I. Frengova, Z. I. Simov, and Z. P. Dimitrov. 2003. Production of volatile aroma compounds by kefir starter cultures. Int. Dairy J. 13:529-535.

Bodyfelt, F. W., and D. Potter. 2008. Creamed cottage cheese. Pages 167-190 in The Sensory Evaluation of Dairy Products. Springer US, New York, NY.

Buttery, R. G., J. D. Turnbaugh, and L. C. Ling. 1988. Contribution of volatiles to rice aroma. J. Agric. Food Chem. 36:1006-1009.

Carballo, J., R. Martin, A. Bernardo, and J. Gonzalez. 1991. Purification, characterization and some properties of diacetyl(acetoin) reductase from Enterobacter aerogenes. Eur. J. Biochem. 198:327332.

Chaves, A. C. S. D., P. Ruas-Madiedo, M. Starrenburg, J. Hugenholtz, and A. L. S. Lerayer. 2011. Impact of engineered Streptococcus thermophilus strains overexpressing glyA gene on folic acid and acetaldehyde production in fermented milk. Braz. J. Microbiol. 34(Suppl. 1):114-117.

Cheng, H. 2010. Volatile flavor compounds in yogurt: A review. Crit. Rev. Food Sci. Nutr. 50:938-950.

Collins, E. B. 1972. Biosynthesis of flavor compounds by microorganisms. J. Dairy Sci. 55:1022-1028.

Condurso, C., A. Verzera, V. Romeo, M. Ziino, and F. Conte. 2008. Solid-phase microextraction and gas chromatography mass spectrometry analysis of dairy product volatiles for the determination of shelf-life. Int. Dairy J. 18:819-825.

Corcoran, B. M., C. Stanton, G. Fitzgerald, and R. P. Ross. 2005. Survival of probiotic Lactobacilli in acidic environments is enhanced in the presence of metabolizable sugars. Appl. Environ. Microbiol. 71:3060-3067.

Corral, S., E. Leitner, B. Siegmund, and M. Flores. 2016. Determination of sulfur and nitrogen compounds during the processing of dry fermented sausages and their relation to amino acid generation. Food Chem. 190:657-664.

de Vos, W. M. D., and J. Hugenholtz. 2004. Engineering metabolic highways in lactococci and other lactic acid bacteria. Trends Biotechnol. 22:72-79.

Dumont, J. P., S. Roger, and J. Adda. 1974a. Etude des composés volatils neutres présents dans les fromages à pâte molle et à croûte lavée. Dairy Sci. Technol. 54:31-43.

Dumont, J. P., S. Roger, P. Cerf, and J. Adda. 1974b. Etude des composés neutres volatils présents dans le Camembert. Dairy Sci. Technol. 54:501-516.

Erkus, O., B. Okuklu, A. F. Yenidunya, and S. Harsa. 2013. High genetic and phenotypic variability of Streptococcus thermophilus strains isolated from artisanal Yuruk yoghurts. Lebensm. Wiss. Technol. 58:348-354.

Gaafar, A. M. 2007. Volatile flavor compounds of yoghurt. Int. J. Food Sci. Technol. 27:87-91.

Georgala, A. K., E. Tsakalidou, I. Kandarakis, and G. Kalantzopoulos. 1995. Flavor production in ewe's milk and ewe's milk yoghurt, by single strains and combinations of Streptococcus thermophilus and Lactobacillus delbrueckii ssp. bulgaricus, isolated from traditional Greek yoghurt. Lait 75:271-283.

Gezginc, Y., F. Topcal, S. Comertpay, and I. Akyol. 2015. Quantitative analysis of the lactic acid and acetaldehyde produced by Streptococcus thermophilus, and Lactobacillus bulgaricus, strains isolated from traditional Turkish yogurts using HPLC. J. Dairy Sci. 98:1426-1434.

Guler, M. O., and S. I. Stupp. 2007. A self-assembled nanofiber catalyst for ester hydrolysis. J. Am. Chem. Soc. 129:12082-12083.

Herve-Jimenez, L., I. Guillouard, E. Guedon, S. Boudebbouze, P. Hols, V. Monnet, E. Maguin, and F. Rul. 2009. Postgenomic analysis of Streptococcus thermophilus cocultivated in milk with Lactobacillus delbrueckii ssp. bulgaricus: Involvement of nitrogen, purine, and iron metabolism. Appl. Environ. Microbiol. 75:2062-2073.

Kaneko, D., T. Igarashi, and K. Aoyama. 2014. Reduction of the offflavor volatile generated by the yogurt starter culture including 
Streptococcus thermophilus and Lactobacillus delbrueckii ssp. bulgaricus in soymilk. J. Agric. Food Chem. 62:1658-1663.

Margalith, P. Z. 1981. Dairy products. Page 32 in Flavor Microbiology. C. C. Thomas, Springfield, IL.

Muramalla, T., and K. J. Aryana. 2011. Some low homogenization pressures improve certain probiotic characteristics of yogurt culture bacteria and Lactobacillus acidophilus LA -K. J. Dairy Sci. 94:3725-3738.

Nieto-Arribas, P., S. Seseña, J. M. Poveda, R. Chicón, L. Cabezas, and L. Palop. 2011. Enterococcus populations in artisanal Manchego cheese: Biodiversity, technological and safety aspects. Food Microbiol. 28:891-899.

Ning, L., F. P. Zheng, H. T. Chen, S. Y. Liu, C. Gu, Z. Y. Song, and B. G. Sun. 2011. Identification of volatile components in Chinese Sinkiang fermented camel milk using SAFE, SDE, and HS-SPMEGC/MS. Food Chem. 129:1242-1252.

Ongol, M. P., Y. Sawatari, Y. Ebina, T. Sone, M. Tanaka, F. Tomita, A. Yokota, and K. Asano. 2007. Yoghurt fermented by Lactobacillus ssp. bulgaricus $\mathrm{H}^{+}$-ATPase-defective mutants exhibits enhanced viability of Bifidobacterium breve during storage. Int. J. Food Microbiol. 116:358-366.

Ott, A., L. B. Fay, and A. Chaintreau. 1997. Determination and origin of the aroma impact compounds of yogurt flavor. J. Agric. Food Chem. 45:850-858.

Pan, D. D., Z. Wu, T. Peng, X. Q. Zeng, and H. Li. 2014. Volatile organic compounds profile during milk fermentation by Lactobacillus pentosus and correlations between volatiles flavor and carbohydrate metabolism. J. Dairy Sci. 97:624-631.

Panagiotidis, P., C. Tzia, A. M. Spanier, F. Shahidi, T. H. Parliment, and C. Mussinan. 2001. Effect of milk composition and heating on flavor and aroma of yogurt. Pages 160-167 in Food Flavors and Chemistry: Advances of the New Millennium. Royal Soc. Chem., Cambridge, UK.

Panighel, A., and R. Flamini. 2014. Applications of solid-phase microextraction and gas chromatography/mass spectrometry (SPMEGC/MS) in the study of grape and wine volatile compounds. Molecules 19:21291-21309.
Pereda, J., D. P. Jaramillo, J. M. Quevedo, V. Ferragut, B. Guamis, and A. J. Trujilo. 2008. Characterization of volatile compounds in ultra-high-pressure homogenized milk. Int. Dairy J. 18:826-834.

Pionnier, E., and D. Hugelshofer. 2006. Characterisation of key odorant compounds in creams from different origins with distinct flavors. Dev. Food Sci. 43:233-236.

Pogačić, T., M. B. Maillard, A. Leclerc, C. Hervé, V. Chuat, F. Valence, and A. Thierry. 2016. Lactobacillus, and Leuconostoc, volatilomes in cheese conditions. Appl. Microbiol. Biotechnol. 100:2335-2346.

Rascón-Díaz, M. P., J. M. Tejero, P. G. Mendoza-Garcia, H. S. García and M. A. Salgado-Cervantes. 2010. Spray drying yogurt incorporating hydrocolloids: Structural analysis, acetaldehyde content, viable bacteria, and rheological properties. Food Bioproc. Techol. 5:560-567.

Rattray, F. P., D. Myling-Petersen, D. Larsen, and D. Nilsson. 2003. Plasmid-encoded diacetyl (acetoin) reductase in Leuconostoc pseudomesenteroides. Appl. Environ. Microbiol. 69:304-311.

Settachaimongkon, S., M. J. Nout, E. C. Antunes Fernandes, K. A Hettinga, J. M. Vervoort, T. C. van Hooijdonk, M. H. Zwietering, E. J. Smid, and H. J. van Valenberg. 2014. Influence of different proteolytic strains of Streptococcus thermophilus in co -culture with Lactobacillus delbrueckii ssp. bulgaricus on the metabolite profile of set-yoghurt. Int. J. Food Microbiol. 177:29-36.

Siek, T. J., I. A. Albin, L. A. Sather, and R. C. Lindsay. 2006. Taste thresholds of butter volatiles in deodorized butteroil medium. J. Food Sci. 34:265.

Starrenburg, M. J., and J. Hugenholtz. 1991. Citrate fermentation by Lactococcus and Leuconostoc spp. Appl. Environ. Microbiol. 57:3535-3540.

Zha, M., J. Yu, Y. Zhang, H. Wang, N. Bai, Y. Qin, D. Liangliang, W. Liu, H. Zhang, and M. Bilige. 2015. Study on Streptococcus thermophilus isolated from Qula and associated characteristic of acetaldehyde and diacetyl in their fermented milk. J. Gen. Appl. Microbiol. 61:50-56. 